\title{
Legal Relief for Performance Obstacles of House Leasing Contract Caused by COVID-19
}

\section{Ran Luo}

Law School of Beijing Normal University, Beijing, China

\begin{abstract}
COVID-19 and its control measures have caused obstacles to the performance of many house-leasing contracts. To give reasonable relief to contracts' Parties involved, the legal nature of COVID-19 and its control measures must be precisely defined. This paper argues that the early COVID-19 and its control measures are the force majeure, and the legal nature of COVID-19 and its control measures under the normal epidemic preventing and controlling mechanism should be analyzed in light of the specific situation. We should accurately grasp the meaning of impossibility of performance, frustration of purpose and impracticability, appropriately take the legal relief, balance the interests of both Parties, and promote social and economic recovery finally.
\end{abstract}

Keywords: force majeure; principle of changed circumstances; impossibility of performance; frustration of purpose; impracticability

\section{Introduction}

At the beginning of 2020, COVID-19 swept the world. It had a bigger impact than SARS in 2003. In order to effectively control the COVID-19 and ensure the safety and health of people, governments of various countries have introduced hierarchical control measures, which have brought about a series of impacts on economic and social life, among which the performance obstacles of continuing contracts are particularly prominent. As a typical continuing contract, house-leasing contract is closely related to people's life and enterprises' development. How to carry on the appropriate legal relief for the obstacles of the performance of the house-leasing contract is not only related to the balance of interests between the Parties of the contract, but also related to the recovery of social economy and the development of small and micro enterprises.

The obstacles of the contract performance mainly include impossibility of performance, frustration of purpose and impracticability. Although China's Contract Law, The Interpretation II of the Supreme People's Court on Certain Issues concerning the Application of the Contract Law of the People's Republic of China, Guidance I on The Proper Trial of Civil Cases involving COVID-19 in Accordance with Law (hereinafter referred to as Guidance I), Guidance II on The Proper Trial of Civil Cases involving COVID-19 in Accordance with Law (hereinafter referred to as Guidance II), and Guidance III on The Proper Trial of Civil Cases involving COVID-19 in Accordance with Law (hereinafter referred to as Guidance III), have made relevant provisions on contract performance obstacles caused by COVID-19, including special provisions on the house-leasing contract, there are still great disputes about the legal relief for the performance obstacles of 
the house-leasing contract. First of all, people have different views on the legal nature of COVID-19 and its control measures, which determines the different ways of legal relief. Secondly, there are disputes about the legal relief of different types of contract performance obstacles. For example, what are the cases of the impossibility of performance? Whether the legal relief of frustration of purpose is limited to the exemption of liability for breach of the contract? If a Party of the contract requests for a change in the contract, what criteria should the court use for judgment?

\section{The Legal Nature of COVID-19 and Its Control Measures}

2.1 The legal nature of the early COVID-19 and its control measures

At the early stage of the outbreak of COVID-19, people did not know enough about its transmission mode, prevention means and treatment methods. People can't make effective prejudgment of COVID-19 and its control measures. In the case of COVID-19 in China, the period during which local governments initiated a level 1st response to a major public health emergency and reduced it to a level 3rd response to a major public health emergency should be an early stage of COVID19. At present, the discussions of the legal nature of COVID-19 and its control measures in China are basically around the early stage of COVID-19. And there are mainly two views.

One view holds that it is force majeure, mainly based on the following reasons. First of all, according to paragraph 2 of Article 117 of the Contract Law of the People's Republic of China, "Force majeure refers to unforeseeable, unavoidable and insurmountable objective conditions". COVID-19 and its control measures meet the above characteristics. Secondly, from the relevant replies from the Legal Affairs Committee of the National People's Congress and the judicial policies of local courts, they all believe that COVID-19 and its control measures are force majeure. Thirdly, during the SARS period, the Supreme People's Court recognized the SARS and its control measures as force majeure. This COVID-19 epidemic is essentially the same as the SARS, so it should make the same determination.

The other view is that it is necessary to distinguish the nature of the epidemic and its control measures. Sometimes it is the force majeure and force majeure rules should be applied. Sometimes it is a change of circumstances and rules of changed circumstances should be applied. Those who hold this view are mainly based on the following reasons. First of all, the force majeure and changed circumstances overlap. It is meaningless to simply discuss the legal nature of COVID-19 and its control measures. The nature should be determined based on its impact on the contract performance obstacles. If the contract cannot be performed due to the COVID-19 and its control measures, it will be deemed as the force majeure. If it makes the performance of the contract difficult, it is the changed circumstance. Secondly, the Legal Affairs Committee of the National People's Congress only puts the situation of impossibility of the contract performance under the category of force majeure. It was vague about what made the impracticability of the contract performance. Thirdly, in the supreme People's Court's judicial interpretation of the SARS, both force majeure and circumstances have room for application. The Supreme People's Court did not give a very clear opinion.

On the whole, I agrees with the first view. In the first place, COVID-19 and its control measures meet the elements of the force majeure. The Contract Law of our country has made the law stipulation about the force majeure. The dominant ideology figures that the concept of the force majeure in Chinese law adopts the compromise theory which holds the view that constituting the force majeure should meet five requirements. Firstly, the force majeure is an objective condition rather than human behaviour. Secondly, the debtor cannot foresee such an objective condition. Thirdly, the debtor cannot avoid the objective condition. Fourthly, the debtor cannot overcome this objective condition. Fifthly, the above-mentioned elements must exist simultaneously. By reviewing of COVID-19 and its control measures, which began on 24 January 2020 , it undoubtedly meets the requirements of force majeure. In the second place, the force majeure and changed circumstances are causal relations. The principle of changed circumstances refers to that after the contract is effectively 
established, if the basis of the contract is shaken or lost due to unforeseen events of the Parties and it is contrary to good faith (obviously unfair) to continue to maintain the original effect of the contract, the contract shall be allowed to change or terminate. There are many reasons why the principle of changed circumstances may apply, and it is obvious that the force majeure may also cause the foundation of a contract to be shaken or lost. Therefore, the force majeure and the principle of changed circumstances can be regarded as the relationship between cause and effect. Failure to perform the contract due to the force majeure may result in the effect of rescission of the contract (pursuant to the Paragraph 1 of Article 94 of China's Contract Law) or the effect of exemption of liability (pursuant to Paragraph 1 of Article 117 of China's Contract Law). And if the performance is difficult due to the force majeure and the continued performance of the contract is obviously unfair or the purpose is not achieved, the principle of changed circumstances may be applied to result in the effect of modification or termination of the contract. Although article 26 of China's Interpretation (II) of Contract Law excludes the force majeure from the principle of changed circumstances, it is not strictly observed in judicial practice. Some judgments affirms the application of the force majeure to the principle of changed circumstances. The mainstream view of the theoretical circle also thinks that the Article 26 of China's Interpretation (II) of Contract Law improperly narrows the scope of application of the changed circumstances, and the force majeure should be included in the scope of application of the principle of changed circumstances. This mainstream view is also adopted in Article 533 of China's Civil Code. Therefore, COVID-19 and its control measures, which are in different dimensions, should not be regarded as changed circumstances on the grounds of applying the principle of changed circumstances.

2.2 The legal nature of COVID-19 and its control measures under the normal epidemic preventing and controlling mechanism

With the human understanding of COVID-19 deepening, the preventing and controlling effect has been getting better. Take China as an example, local governments have adjusted the preventing and controlling level of COVID-19, established regular preventing and controlling plans, issued the negative list, and put forward preventing and controlling requirements for various industries. The potential containment measures become clearer. COVID-19 and its control measures, such as "COVID-19 will coexist with humanity for a certain period of time", "Dense population areas are high risk areas", "The specific content of the first-level response, second-level response and third-level response" and so on, are not unforeseen. Instead, people can already predict the possible outcome. Under such circumstances, it is no longer appropriate to treat COVID-19 and its control measures as the force majeure and they should be treated in a categorized manner:

Firstly, COVID-19 and its control measures within the foreseeable scope are no longer the force majeure. It has become the consensus of the whole society that COVID-19 will exist for quite a long time and human beings cannot completely eliminate it in a short time. At present, people are familiar with control measures of the government and even can predict them when COVID-19 outbursts again. Therefore, under the normal epidemic preventing and controlling mechanism, if there is no special provision in the contract, COVID-19 and its control measures are no longer meet the requirements of the force majeure. Its repeated and local outbreak is not the force majeure, it is more appropriate to identify it as the commercial risk.

Secondly, for unforeseen factors of COVID-19 and its control measures, such as the sudden changes of COVID-19, new control measures and so on, if they meet the requirements of the force majeure, they should still be considered as the force majeure. The COVID-19 may mutate, its speed of transmission and its impact on human health may change, and people's understanding of it is still not comprehensive, so the corresponding government control measures will be adjusted accordingly. At the moment, the elements that meet the requirements of the force majeure shall be recognized as the force majeure, while those that meet the requirements of other legal facts shall be recognized as other legal facts. 


\section{Legal Remedies for the Impossibility of Performance}

3.1 The meaning of impossibility of performance

The impossibility of performance refers to the impossibility to complete the payment of the contract's object after the contract becomes effective, including the impossibility in law and the impossibility in fact. In general, economic impossibility is not impossibility of performance; it is impracticability. In terms of the house-leasing contract, if the house as the subject matter of the contract is damaged due to the earthquake, debris flow or other force majeure and the lessor is unable to perform the obligation of delivering the house, it is impossibility of performance. However, if the tenant who is no business income to pay the rent, which is caused by control measures of the government, it is not impossibility of performance. It is impracticability.

\subsection{Relevant legal remedies}

There are two views on the legal effect of impossibility of performance due to the force majeure. In the first view, the effect is exemption of the liability. The reason is that according to the first paragraph of Article 117 of the Contract Law and the first paragraph of Article 3 of Guidance Opinion I, if the force majeure causes the debtor to be unable to perform the contract, it can be exempted from liability in whole or in part. The reason is that according to the paragraph 1 of Article 117 of the Contract Law and the paragraph 1 of Article 3 of Guidance I, if the force majeure causes the debtor to be unable to perform the contract, it can be exempted from liability partly or overall. The second view holds that in addition to the legal effect of exempting the debtor, the Parties may also terminate the contract. The reason is that although the paragraph 1 of Article 94 of the Contract Law stipulates that a contract can be terminated only if the purpose of the contract cannot be achieved due to the force majeure, the fundamental reason for the termination of the contract is impossibility of performance.

Fundamentally speaking, there is little difference between the two viewpoints. Impossibility of performance is the premise that the purpose of contract cannot be achieved. However, according to the provisions of Article 533 of the Civil Code on the change of circumstances, this paper believes that the first viewpoint is more preferable. On the one hand, the provision about the changed circumstances in Civil Code, compared with the Article 26 of Interpretation II of Contract Law, excludes the situation that the purpose of the contract cannot be achieved, and only relieve the situation of obvious injustice of the contract. If the second view is adopted, the Parties may terminate the contract when the contract cannot performed due to the force majeure. It excludes the circumstances that the purpose of the contract cannot be achieved due to the force majeure. Of course, the Party may exercise the right of termination according to other legal provisions, but it is not as clear and feasible as the direct provision. On the other hand, impossibility of performance is the reason why the typical purpose of a contract is not achieved. Adding a chain of results does not make much difference of the legal effect. And it is the legislative succession for many years, there is no compelling reason to change.

\section{Legal Remedies for Frustration of Purpose}

4.1 The meaning of frustration of purpose

The frustration of purpose means that the purpose of the contract cannot be achieved. The purpose of contract includes two kinds: the typical purpose and the subjective purpose. The typical purpose is objective, which can be directly obtained by the Parties through the performance of the contract. In the same kind of the contract, the Parties have the same purpose. The typical purpose of a contract can be achieved as long as the contract is performed as agreed. For example, in the house-leasing contract, the lessor's typical contract purpose is to obtain the rent, and the tenant's typical contract purpose is to obtain the right to use the house. The subjective purpose can be understood as the contractual motive meeting the certain criterion. It is the indirect effect that the Parties intend to achieve through the performance of the contract. Not 
all the contract motives can become the purpose of a contract. Only when both Parties know or should know the motive, and the motive constitutes the basis of the contract's forming, can it be identified as the purpose of a contract. Still taking the house-leasing contract, for example, in the case of Krell V. Henry, the tenant rented a viewing space for the British Royal Parade. Later, the parade was canceled. The tenant requested to terminate the contract because he believed that the purpose of the contract could not be achieved. The court upheld his claim. In this case, the tenant used its contractual motive as the purpose of its contract.

\subsection{Relevant legal remedies}

If the purpose of the contract is not achieved due to the force majeure, the Parties may terminate the contract, which is a cause recognized by most countries, including China. Influenced by COVID-19 and its control measures, many Parties of the house-leasing contract ask to terminate the contract. For the lessor, the purpose of the contract is to collect the rent. If the tenant does not pay the rent, does the lessor have the right to terminate the contract? According to Article 5 of Guidance II, "If the lessor meets capital turnover difficulties or significant decrease in operating income because of COVID-19 and its control measures so that cannot pay the rent, the people's court shall not support the lessor's request of terminating the lease contract and asking the tenant to take the liability of breaking the contract." It means that for the operating house, the lessor cannot terminate the contract because the tenant has difficulty in operating and fails to pay the rent. This paper agrees the view. COVID-19 and its control measures have caused difficulties for the tenant, and it is obviously unfair to continue to perform the original contract. This situation is the changed circumstances. It should be negotiated by both Parties at first, and one Party cannot exercise the right of rescission directly. Moreover, the tenant's failure to perform a period of rent may not seriously affect the economic benefits expected by the lessor, and may not result in the failure to achieve the purpose of the contract.

For the legal relief of both Parties under the changed circumstances, this paper will be discussed in detail in the fifth part. What is explained here is that after the outbreak of COVID-19 and its control measures, if the two Parties reach a new agreement to change the original contract content and the tenant fails to pay the rent to a certain extent, the lessor shall be entitled to the right to terminate the contract, and the tenant can be held liable for breach of contract. On the one hand, the modified contract has changed the unfair situation of the original contract and rebalanced the interests of both Parties, so it is legitimate to perform the contract. On the other hand, the contract change occurs after COVID-19 and its control measures. At this time, COVID-19 and its control measures are not unpredictable and do not belong to the force majeure. Therefore, the lessor has the right to investigate the tenant's liability for breach of contract, and has the right to terminate the contract after meeting certain conditions.

If the tenant is unable to use the house for business due to COVID-19 and its control measures, then the purpose of the contract can not achieved, does the tenant have the right to terminate the contract? This should be handled separately. For the house-leasing contract based on a specific purpose, if the purpose can not achieved due to the force majeure, the tenant has the right to terminate the contract. The same is true of the Paragraph 2, Article 5 of Guidance II. For the tenant without subjective purpose, we should combine with the leasing period to determine whether the purpose of the contract can not be achieved. If the remaining leasing term is short, the tenant may terminate the contract. If the remaining leasing term is longer, then generally the tenant should not be entitled the right to terminate the contract. It should be noted that although the tenant does not have the right to terminate the contract, the loss of the tenant resulting from the failure of typical purposes may be shared by both Parties. For example, if the tenant is stranded outside and cannot return to the rented house due to COVID-19 and its control measures, the lessor can be requested to reduce the rent or extend the leasing term appropriately. At the same time, it should be noted that if both Parties agree to change the contract after 
COVID-19 and its control measures, the tenant is not entitled to terminate the contract under the estoppel rule in general.

\section{Legal Remedies for Impracticability}

COVID-19 and its control measures have brought a great impact on catering, entertainment and educational institutions, etc. In practice, many tenants has difficulties in performing. Many influential catering enterprises are also facing greater cash flow pressure. As mentioned above, such situation is the changed circumstances and shall be handled in accordance with the principle of changed circumstances. The Parties may request the people's court to apply the principle of fairness to modify the contract. According to Article 6 of Guideline II, the Supreme People's Court also made provisions according to this idea, and made special provisions on state-owned houses based on the current situation of state-owned houses and the purpose of protecting small, medium and micro enterprises. The following points should be noted with regard to Article 6:

Firstly, the tenant leases the house for business purposes, which means that this Article excludes the application of the residential house-leasing contract. Some people believe that this is because the function of residential house is to live, which is less affected by COVID-19 and its control measures, and generally within the risk range that the tenant can bear.

Secondly, the tenant must have a difficult situation to perform, such as "operating difficulties", "no business revenue or significant decrease of the business revenue", and so on, and the difficult situation is due to COVID-19 and its control measures.

Thirdly, we should treat state-owned and non-state-owned houses differently. For state-owned houses, the house's owner will reduce or exempt the rent, according to national policy, as long as the tenant have a difficult to fulfil the contract, no matter whether the continued performance of the contract resulted in an obvious injustice or not. For the nonstate-owned houses, on the one hand, the lessor should have the typical elements of the changed circumstances, and meet the difficult performance and obvious injustice due to continued performance; on the other hand, the court generally mediate in accordance with the national policy, or judge according to the principle of fairness when the mediation fails.

5.1 Whether the non-operating house-leasing contract can apply the principle of changed circumstances

The fact that Article 6 of Guidance II does not include non-operating houses in its prescribed scope does not mean that the principle of changed circumstances is not applicable to such houses. First of all, although the main function of nonoperating housing is its use function, it does not mean that it is less affected by COVID-19 and its control measures. For example, if a foreigner living in the epidemic area is unable to return to the local area due to the "closure of the city" and other control measures, the local rented houses will not be able to play their functions. If the tenant cannot get the rent reduction for the locally rented premises, then the tenant has to pay the full rent for both premises. It's obviously unfair. Secondly, the individuals' financial strength and anti-risk ability are often lower than enterprises, so the view that residential houses' rent belongs to the risk that individuals can bear is groundless.

\subsection{How to grasp the principle of fairness}

The biggest problem of the application of the fairness principle in judgment is its ambiguity and uncertainty. From the Guideline II, country's relevant policies seem to be a relatively clear standard. However, on the one hand, the national policy is only applicable to the contract modification of state-owned houses; on the other hand, some of the policies have not set clear standards. In many cases, judges are still required to exercise their discretion. So it is very important to discuss the standard of changing the house-leasing contract.

\subsubsection{The existing standards for judging}

(1) Rent reduction policies of governments shall be directly applied

At present, there are many differences of the central government's policy and the local government's policy, such as 
the target of reduction or exemption, the period of reduction or exemption, and so on. In accordance with the Guidance on Coping with COVID-19 to Help Small and Micro Businesses and Individual Businesses in the Service Industry to Alleviate Rent Pressure, issued by the National Development and Reform Commission, Ministry of Housing and Urban-Rural Development, Ministry of Finance, Ministry of Commerce, People's Bank of China, Sasac, State Administration of Taxation and State Administration of Market Regulation, the owners of state-owned houses should exempt small and micro businesses and individual businesses who have business difficulties and rent houses for business in the Service Industry from paying the rent for three months in the first half of the year. In addition to implementing the central government's guidance, local governments have also made other rent reduction and exemption policies. For example, in accordance with the Several Measures to Deal with COVID-19 and Promote the Sustainable and Healthy Development of Micro, Small and Medium-sized Enterprises, issued by the People's Government of Beijing, the rent reduction and exemption are applicable to small, medium and micro enterprises in all industries, and are not limited to operating houses. But the intensity of relief is different, for the rental housing for business, the rent for February is exempted. And for tenants who rent the houses for office use, $50 \%$ of the rent for February will be exempted. In accordance with the Notice on the Issuance of Policies and Measures to Control COVID-19 and Support the Steady and Healthy Development of Service Enterprises in Shanghai, issued by the People's Government of Shanghai, is also only applicable to service industry enterprises who rent houses for business. But it is not only suitable for small and micro enterprises, but also suitable for medium-sized enterprises. In accordance with the Twenty Policy Measures to Deal with COVID-19 to Support Small and Medium-sized Enterprises in Weathering the Storm Together, issued by Chongqing Municipal People's Government General Office, the small, medium and micro enterprises that rent the operating houses of state-owned assets, whether they belong to service enterprises or not, will be reduced or exempted the rent for one to three months. However, the document did not specify how to determine the duration of relief.

(2) Considering all relevant factors

Some courts' judgments do not list the basis of judgment specifically, but refer to the comprehensive consideration of various circumstances. For example, in the case of "Cheng Meiping and Rao Jun's house-leasing contract dispute" the judgment of the second-instance court said, “...... Considering the actual situation of this case, the extent to which Fengjie county and the catering and accommodation industry have been affected by COVID-19....."

(3) Paying the rent according to the proportion of the house area available

The US bankruptcy court took this approach in the HITZ Restaurant Group case. During the epidemic, HITZ Catering Group could not receive guests in its business premises due to the administrative order, and could only carry out food delivery and other businesses, which affected its solvency. The court granted rent relief to the HITZ Restaurant Group, requiring it to pay the appropriate rent, based on the proportion of the actual usable house area in the total leased house area, in this case $25 \%$.

All of the above methods have their rationality, and the government's policies are targeted. They are formulated in combination with the impact of the epidemic and its control measures in the country or the region, and based on specific policy purposes. They are a manifestation of the state's macro-control power. The comprehensive consideration approach focuses on the measurement of benefits in individual cases. That according to the ratio of actual usable house area to determine the amount of rent relief also reflects the simple concept of fairness. However, these methods all have their shortcomings. The government's rent reduction and exemption policies usually target at state-owned houses, and the same standard for non-state-owned houses may lead to "acclimatization". At the same time, as mentioned earlier, some local policies are not definitive and do not specify the specific amount of relief. In some cases, it may be fair to determine the 
amount of rent relief based on the proportion of actual usable house area, but in some cases, it cannot balance the interests of the Parties. The method of comprehensive consideration is too vague and the discretion of the judge is too great.

\subsubsection{Solution path}

The purpose of applying the principle of changed circumstances to a contract is to return the rights and obligations of the Parties to a fair and balanced state. That's our starting point for changing the contract, so what's fair? After the development of human civilization for thousands of years, the definition of civil justice is that everyone can get the benefits he deserves in the civil field. Fairness is too vague and too difficult to grasp. Specifically, under the situation that the principle of changed circumstances applies to the house-leasing contract, justice of exchange and justice of distribution are the inherent meaning of the fairness principle. From the perspective of comparative law, the "basic theory of transaction" in Germany has gone through a hundred years of development. Although it is still controversial in theory, in judicial practice, judges have integrated all the factors of individual cases to make contracts reach a new state of fairness. The rule of "frustration of the contract's purpose" in common law system is also the affirmation of the value of the principle of changed circumstances, and its reasonable application is also a great test to the wisdom of judges. Generally speaking, both two jurisdictions require strict application of the principle of changed circumstances on the premise of affirming the principle, so as to prevent judges from making arbitrary decisions, which is also enlightening to our country. In the context of COVID-19, applying the principle of changed circumstances to change the house-leasing contract, judges should accurately grasp the principle of fairness. In order to achieve objective equivalence and distributive justice, the following factors should be taken into account:

(1) Room Type

Distributive justice is the determination of each man's share of the advantage and the disadvantage according to geometrical proportions. In the case that COVID-19 has caused obvious unfairness in the house-leasing contract, the main way to achieve distributive justice is to distribute the disadvantage suffered by the tenant. But the house type is different, whether the tenant suffers from disadvantage and the degree of disadvantage is different. Taking office houses as example, in fact, they carry more functions, and COVID-19 may not cause damage to the interests of the tenants. The tenants use the office houses to ensure the normal operation of the headquarters of the enterprise that can timely adjust the enterprise strategy and change the business direction, etc. It can minimize the tenants' loss caused by COVID-19 and its control measures, and tenants' interests in renting the office area are not damaged. Of course, the tenants who rent office houses may have difficulties in performance, but his lease interest is not damaged. In this case, the judge can allow tenants to have a grace period of performance instead of reducing or exempting the rent. For commercial buildings, the tenants may have a significant decrease or no revenue at all due to COVID-19 and its control measures, thus resulting in unfair performance of the contract, and may be allowed to reduce or exempt rent.

(2) Industry

Since the premise of rent reduction for commercial buildings is the reduction of the tenant's operating income, the judge can decide the proportion of rent reduction according to the operating conditions of the tenant's industry. Thus distributing the loss equally among the Parties to the contract. For example, according to China's statistical data, in March 2020, "the income of catering enterprises above the quota decreased by $46.7 \%$ year on year, but the retail revenue of Chinese and Western medicine products above the quota increased by $8.0 \%$ year-on-year." Therefore, for the tenant of catering industry, the tenant can be reduced rent in proportion, which is $23.35 \%$ of the original rent in the contract. But for the tenant of pharmaceutical industry, as its operating condition is not affected, it will not be given rent reduction or exemption. 
(3) Market rents and vacancy rates

The reason why civil law takes subjective equivalence as the general practice of equivalence principle is that the objective equivalence standard is often not determined, and the market price is often a relatively fair standard that is easy to obtain under the market economy background. Compared with the establishment of the contract, the foundation of the contract has been shaken and it is proper to take the market rent as the standard to balance the interests of contract's Parties when the situation changes. Because it can realistically reflect the market value of the leased houses. But it should be noted that sometimes there is a phenomenon of "price without market" (it means there are no customers willing to pay for a higher rental price) in the house-leasing market. Therefore, the vacancy rate of houses should be considered together with the price to make it more close to the requirements of exchange justice.

\section{Conclusions}

The early COVID-19 and its control measures are the force majeure. Under the normal epidemic preventing and controlling mechanism, COVID-19 and its control measures within the foreseeable scope are no longer the force majeure and for unforeseen sudden changes of COVID-19 and control measures, while those that meet the conditions shall still be deemed as the force majeure. There is a causal relationship between the force majeure and changed circumstances. For impossibility of the house-leasing contract's performance due to the force majeure, the debtor may be exempted from liability. For frustration of the house-leasing contract's purpose due to the force majeure, the Parties may terminate the contract. For the house-leasing contract's impracticability due to the force majeure, the principle of changed circumstances shall be applied. At this time, if the court changes the contract content in accordance with the principle of fairness, the judge should balance the interests between the Parties by combining the three factors of the room type, the tenant's business, the market rent and the vacancy rate.

\section{Conflicts of Interest}

The author declares no conflicts of interest regarding the publication of this paper.

\section{References}

[1] PhD candidate. Law School. Beijing Normal University.

[2] See F.L. (2020). The Nature and Judicial Response of COVID-19 and Administrative Actions that Constitute the Performance Obstacles of the House-leasing Contract. Legal Application, (5): 43.

[3] Han S.Y. (2018). General Theory of Contract Law (3rd edition). Law Press, Beijing.

[4] Chen H.B. (2012). General Theory of Debt Law (1st edition). China Legal Publishing House, Beijing.

[5] Liu K.X. (2020). Comments and Suggestions for Improvement of Contract Termination System in Civil Code. Tsinghua Law, 3(14): 159.

[6] Cui J.Y. (2015). On the Purpose of Contract and its Frustration. Journal of Social Science of Jilin University, 3(55): 41.

[8] Yu M.Z. (2020). See "Cheng Maiping and Rao Jun Housing Lease Contract Dispute Second Instance Civil Judgment". https://pkulaw.com/pfnl/a6bdb3332ec0adc443857436a762a8f6d71fb15348cce13abdfb.html

[9] See See In Re: Hitz Restaurant Group, B.R. 3 (United States Bankruptcy Court, N.D. Illinois, Eastern Division. 2020).

[10] Yi J. (2012). A New Interpretation of the Principle of Fairness in Civil Law. Jurist, (4): 58-69.

[11] National Bureau of Statistics. (2020). The Total Retail Sales of Consumer Goods Fell by $15.8 \%$ in March 2020. http://www.stats.gov.cn/tjsj/zxfb/202004/t20200417_1739331.html 